V. Ozols

Nagoya Math. J.

Vol. 48 (1972), 169-172

\title{
PERIODIC ORBITS OF ISOMETRIC FLOWS
}

\author{
V. OZOLS
}

\section{Introduction}

Let $M$ be a compact $C^{\infty}$ Riemannian manifold, $X$ a Killing vector field on $M$, and $\varphi_{t}$ its 1-parameter group of isometries of $M$. In this, paper, we obtain some basic properties of the set of periodic points of $\varphi_{t}$. We show that the set of least periods is always finite, and the set $P(X, t)$ of points of $M$ having least period $t$ for the vector field $X$ is a totally geodesic submanifold, with possibly non-empty boundary. Moreover, we show there are at least $m$ geometrically distinct closed geodesic orbits of $\varphi_{t}$, where $m$ is the number of least periods which are not integral multiples of any other least period.

\section{Finiteness of least periods}

Let $M$ be a complete Riemannian manifold of dimension $n$. Let $I^{0}(M)$ be the identity component of its isometry group, and $i(M)$ the Lie algebra of $I^{0}(M) . \quad i(M)$ is naturally identified with the Lie algebra of Killing vector fields on $M$, and we will identify an element $X \in i(M)$ with the corresponding Killing vector field. If $X$ is any vector field on $M$, let Zero $(X)=\left\{p \in M \mid X_{p}=0\right\}$. We use Fix $(f)$ for the set of fixed points of a map $f: M \rightarrow M$.

Lemma. Let $X \in i(M)$ and $\varphi_{t}$ its 1-parameter group. For each $p \in M$ there is a neighborhood $U$ of $p$ such that the set of least periods of periodic orbits of $\varphi_{t}$ which intersect $U$ is finite.

Proof. (i) If $p \in \operatorname{Zero}(X)$ then $\left(\varphi_{t}\right)_{*}: T_{p} M \rightarrow T_{p} M$ is a 1-parameter subgroup of the orthogonal subgroup of the orthogonal group of $T_{p} M$, so there is a basis of $T_{p} M$ in which $\left(\varphi_{t}\right)_{*} \mid T_{p} M$ has the form:

$$
\left(\varphi_{t}\right)_{*}=\operatorname{diag}\left\{a_{1}(t), \cdots, a_{k}(t), I_{n-2 k}\right\}
$$

Received November 16, 1971. 
where

$$
a_{i}(t)=\left(\begin{array}{rr}
\cos \alpha_{i} t & \sin \alpha_{i} t \\
-\sin \alpha_{i} t & \cos \alpha_{i} t
\end{array}\right)
$$

$\alpha_{i} \neq 0$ for $i=1, \cdots, k$, and $I_{n-2 k}$ is the $(n-2 k) \times(n-2 k)$ identity matrix. The periodic points of $\varphi_{t}$ in a spherical neighborhood of $p$ correspond, by $\exp _{p}: T_{p} M \rightarrow M$, to periodic vectors in $T_{p} M$ under $\left(\varphi_{t}\right)_{*}$. The least periods of such vectors are the least common multiples of subsets of the set of numbers $\left\{2 \pi / a_{1}, \cdots, 2 \pi / a_{k}\right\}$ and thus are finite in number.

(ii) If $p \notin \operatorname{Zero}(X)$, let $t_{0}$ be the least period of $p$. (Let $t_{0}=+\infty$ if $p$ is not periodic). If $t_{0}=\infty$, then either $p$ lies in a neighborhood of non-periodic points, or there is a sequence of periodic points $p_{i} \rightarrow p$. In the first case we're done, and in the second we replace $p$ by a $p_{i}$ sufficiently close to $p$ so $p$ lies in a convex normal neighborhood of $p_{i}$. Thus we assume $p$ is periodic of least period $0<t_{0}<+\infty$. For each $\varepsilon>0$ let $N_{\varepsilon}=\left\{Y \in T_{p} M \mid Y \perp X_{p}\right.$ and $\left.|Y|<\varepsilon\right\}$. If $\varepsilon$ is sufficiently small, then $\varphi_{t}\left(\exp _{p} N_{s}\right) \cap \varphi_{t}\left(\exp _{p} N_{\varepsilon}\right) \neq \emptyset \quad$ only when $t-t^{\prime} \equiv 0 \quad\left(\bmod t_{0}\right)$, and $\bigcup_{t} \varphi_{t}\left(\exp _{p} N_{\varepsilon}\right)$ is a tubular neighborhood of the closed orbit $\left\{\varphi_{t}(p) \mid t \in R\right\}$. Let $\delta \in\left(0, t_{0} / 2\right)$ be so small that $U=\bigcup_{t}\left\{\varphi_{t}\left(\exp _{p} N_{\epsilon}\right)|| t \mid<\delta\right\}$ is a normal neighborhood of $p$. We may assume the $p_{i}$ we chose to replace $p$ is close enough to $p$ so they both lie in $U$. Now if we put $\left(\varphi_{t_{0}}\right)_{*} \mid T_{p} M$ in the same normal form as in (i), the same argument shows there are only finitely many least periods of periodic points in $U$.

q.e.d.

Remark. It follows from the proof that the number of least periods in each of the neighborhoods $U$ is bounded above by the maximal number possible of least common multiples in a set of [n/2] numbers; namely $2^{[n / 2]}$. This is independent of the choice of $X$, but the neighborhood $U$ does depend on $X$.

COROLlary. If $M$ is compact, the set of least periods of $\varphi_{t}$ is finite.

This corollary thus follows from a simple geometrical argument. One can derive the same result using a theorem of Yang ([4]), which says that a compact Lie group acting differentiably on a compact manifold has only finitely many non-conjugate isotropy subgroups.

From now on, $M$ will always be compact. The Lie algebra $i(M)$ has an $\operatorname{ad}\left(I^{\circ}(M)\right)$-invariant positive definite symmetric bilinear form, and we let $S$ be the unit sphere in $i(M)$ with respect to this form. 
Lemma. There is a number a>0 such that for every $X \in S$ and every isotropy subgroup $G_{p}$, if $0<t_{0}<a$ and $\exp \left(t_{0} X\right)$ is in $G_{p}$, then $\exp (t X) \in G_{p}$ for all $t \in R$.

Proof. $I^{0}(M)$ is a compact Lie group and therefore a compact symmetric space whose geodesics are the translates of 1-parameter subgroup $\exp (t X)$. If we assume $X \in S$ then $t$ is arc-length. Let $U_{\varepsilon}$ be the open ball of radius $\varepsilon$ about 0 in $i(M)$, and assume $\varepsilon>0$ is so small that $\exp \left(U_{\varepsilon}\right)$ lies in a normal neighborhood of $e$ in $I^{0}(M)$. Suppose $0<t_{0}<\varepsilon$ and $\exp \left(t_{0} X\right) \in G_{p}=$ identity component of $G_{p}$. There is a minimizing geodesic $\exp (t Y)$ from $e$ to $\exp \left(t_{0} X\right)$ lying entirely in $G_{p}^{0}$, whose length is less than $\varepsilon\left(\right.$ since $\exp \left(U_{\varepsilon}\right) \cap G_{p}^{0}$ is the $\varepsilon$-ball about $e$ in $\left.G_{p}^{0}\right)$. Now $\exp \mid U_{\varepsilon}$ is $1-1$ so we must have $\exp (t Y)=\exp (t X)$ for all $t$.

If $G_{p}$ is not connected, it has finitely many components and we let $r_{p}=$ distance $\left(e, G_{p}-G_{p}^{0}\right)$. Clearly $r_{p}>0$ since $G_{p}-G_{p}^{0}$ is compact; and $r_{p}$ is constant on the conjugacy class of $G_{p}$ since the metric in $I^{0}(M)$ is invariant by conjugation. There are only finitely many conjugacy classes so $r=\min r_{p}>0$. Then any $0<a<\min (\varepsilon, r)$ satisfies the requirements of the lemma.

q.e.d.

From this lemma we can derive a "uniform" period bounding lemma for Killing vector fields :

COROLLARY. The positive least periods of periodic orbits of 1-parameter groups of isometries are bounded away from zero uniformly if their generators $X$ are taken from $S$.

Proof. A point $p \in M$ is periodic of least period $t_{0}$ for the 1-parameter group $\exp (t X)$ if $\exp \left(t_{0} X\right) \in G_{p}$ but $\exp (t X) \notin G_{p}$ if $0<t<t_{0}$. The number $a$ of the previous lemma is then the required lower bound. q.e.d.

\section{Submanifolds of periodic points}

For each $X \in i(M)-\{0\}$ and each $0<t<\infty$, let $P(X, t)=\{p \in M \mid p$ has least period $t$ for the 1-parameter group $\exp (t X)\}$. Let $P(X, 0)=$ Zero $(X)$, and $P(X, \infty)=$ set of non-periodic points of $\exp (t X)$. Then we know $P(X, t)=\emptyset$ except for a finite subset of $[0, \infty]$. Now assume $X \in i(M)-\{0\}$ is fixed, and $\varphi_{t}$ is its 1-parameter group. It is well-known that for each $0<t<\infty$, Fix $\left(\varphi_{t}\right)$ is a closed totally geodesic submanifold of $M$. 
Let $0<t_{1}<t_{2}<\cdots<t_{N}$ be the positive least periods for $X \in i(M)$. Let $\beta_{1}, \cdots, \beta_{m}$ be the subset of least periods which are not integral multiples of any other least period. Use the notation $t_{i} \mid t_{j}$ if $t_{j}=k t_{i}$ for some integer $k$, and call the $\beta_{i}$ the basic periods for $X$. It is easy to see that for each $i=1, \cdots, N, P\left(X, t_{i}\right)=\operatorname{Fix}\left(\varphi_{t_{i}}\right)-\cup\left\{\operatorname{Fix}\left(\varphi_{t_{j}}\right)\left|t_{j}\right| t_{i}\right\}$ - Zero $(X)$, the union consisting of finitely many closed totally geodesic submanifolds of $\operatorname{Fix}\left(\varphi_{t_{i}}\right)$. Therefore we have:

Proposition. Each $P\left(X, t_{i}\right)$ is a totally geodesic submanifold of $M$ (possibly with a finite number of closed submanifolds deleted).

THEOREM. If the Killing vector field $X$ has $m$ basic periods, there are at least $m$ geometrically distinct smooth closed geodesics on $M$ which are orbits of the 1-parameter group of $X$.

Proof. For each $i=1, \cdots, m, P\left(X, \beta_{i}\right)=\operatorname{Fix}\left(\varphi_{\beta_{i}}\right)-\operatorname{Zero}(X)$. Now Fix $\left(\varphi_{\beta_{i}}\right)$ is a closed totally geodesic submanifold of $M$, and $X$ is tangent to it, so $X$ is Killing vector field on $\operatorname{Fix}\left(\varphi_{\beta_{i}}\right)$. Let $p \in \operatorname{Fix}\left(\varphi_{\beta_{i}}\right)$ be a point at which $|X|$ achieves its maximum. Then $|X|^{2}$ has a critical point at $p$, so ([2]) the orbit of $p$ is a geodesic. The orbit is non-trivial since $p \notin \operatorname{Zero}(X)$.

q.e.d.

Remarks. (1) In fact, we get a closed geodesic for each component of $\operatorname{Fix}\left(\varphi_{\beta_{i}}\right)$.

(2) The same argument as in Kobayashi ([1]), shows that the Euler numbers of the Fix $\left(\varphi_{t_{i}}\right)$ all equal that of $M$.

\section{BIBLIOGRAPHY}

[1] S. Kobayashi, Fixed points of isometries, Nagoya Math. J. 13, pp. 63-68.

[2] V. Ozols, Critical points of the displacement function of an isometry, J. of Differential Geometry 3 No. 4 (1969), pp. 411-432.

[3] - Critical points of the length of a Killing vector field, (to appear).

[ 4 ] Yang, C. T., On a problem of Montgomery, Proc. Amer. Math. Soc., 8 (1957), 255-257. 